Irish Math. Soc. Bulletin

Number 72, Winter 2013, 75-77

ISSN 0791-5578

\title{
MACCOOL'S SECOND PROOF OF MORLEY'S MIRACLE
}

\author{
M.R.F. SMYTH \\ In memory of Kenneth Beales and Trevor West
}

\begin{abstract}
Here is a traditional proof of Morley's miracle that is unrivalled for brevity and simplicity. It stems from a sadly neglected mathematical gem published in 1914.
\end{abstract}

\section{INTRODUCTION}

That the triangle $X Y Z$ in the figure below is always equilateral is formally known as Morley's trisector theorem and informally as Morley's miracle. Its modern discovery dates back to 1899 and since then it has been proved many times by a wide variety of methods. The website [1] tracks developments and plays host to roughly twenty proofs including MacCool's original effort.

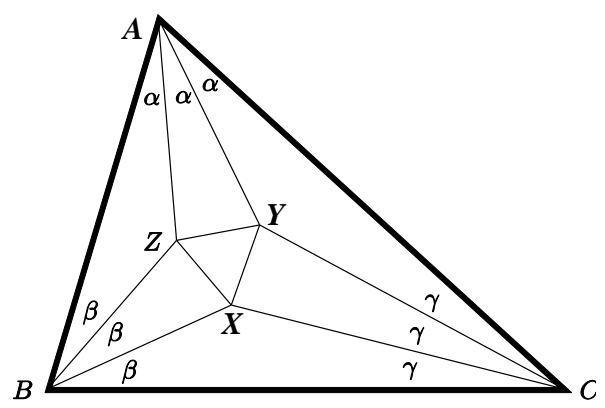

However as [3] explained, the proof there was based entirely on straight line geometry and similar triangles. It opined that MacCool's second notebook which was marked "Advanced" and contained diagrams of circles might hold an alternative proof. And so indeed it has proved, although it has taken me a very long time to decipher the Ogham. So whilst I have yet to find any evidence that MacCool was familiar with Pythagoras, the result that we know today as the inscribed angle theorem [Euclid: Book 3, Prop 22] does

2010 Mathematics Subject Classification. 51M04.

Key words and phrases. Morley, trisector.

Received on 17-8-2013; revised 9-12-2013.

(C)2013 Irish Mathematical Society 
indeed appear in his "Advanced" notebook, and soon afterwards comes the second proof of Morley's theorem. This is even shorter and easier than his "Basic" one, and completely debunks the urban myth that all purely geometric proofs must necessarily be longer and more complex than the "backward" ones.

\section{PROOF}

His proof runs as follows. In any triangle $A B C$ let $X$ be the Morley vertex adjacent to $B C$. First construct the points $P$ and $Q$ on $A B$ and $A C$ respectively such that $|B P|=|B X|$ and $|C Q|=|C X|$. Then construct the right-angled triangle $P R X$ with hypotenuse $P X$ and $\angle X P R=30^{\circ}$ as shown below. The six marked segments will all have equal length. Produce $P R$ and the trisector $C S$ to meet in $Y$. Note that the three right-angled triangles, $\triangle R X Y$ and $\triangle S X Y$ and $\triangle S Q Y$, (which MacCool calls wedges) have equal hypotenuses and an equal (marked) side therefore they are congruent. Evidently $\alpha+\beta+\gamma=60^{\circ}$.

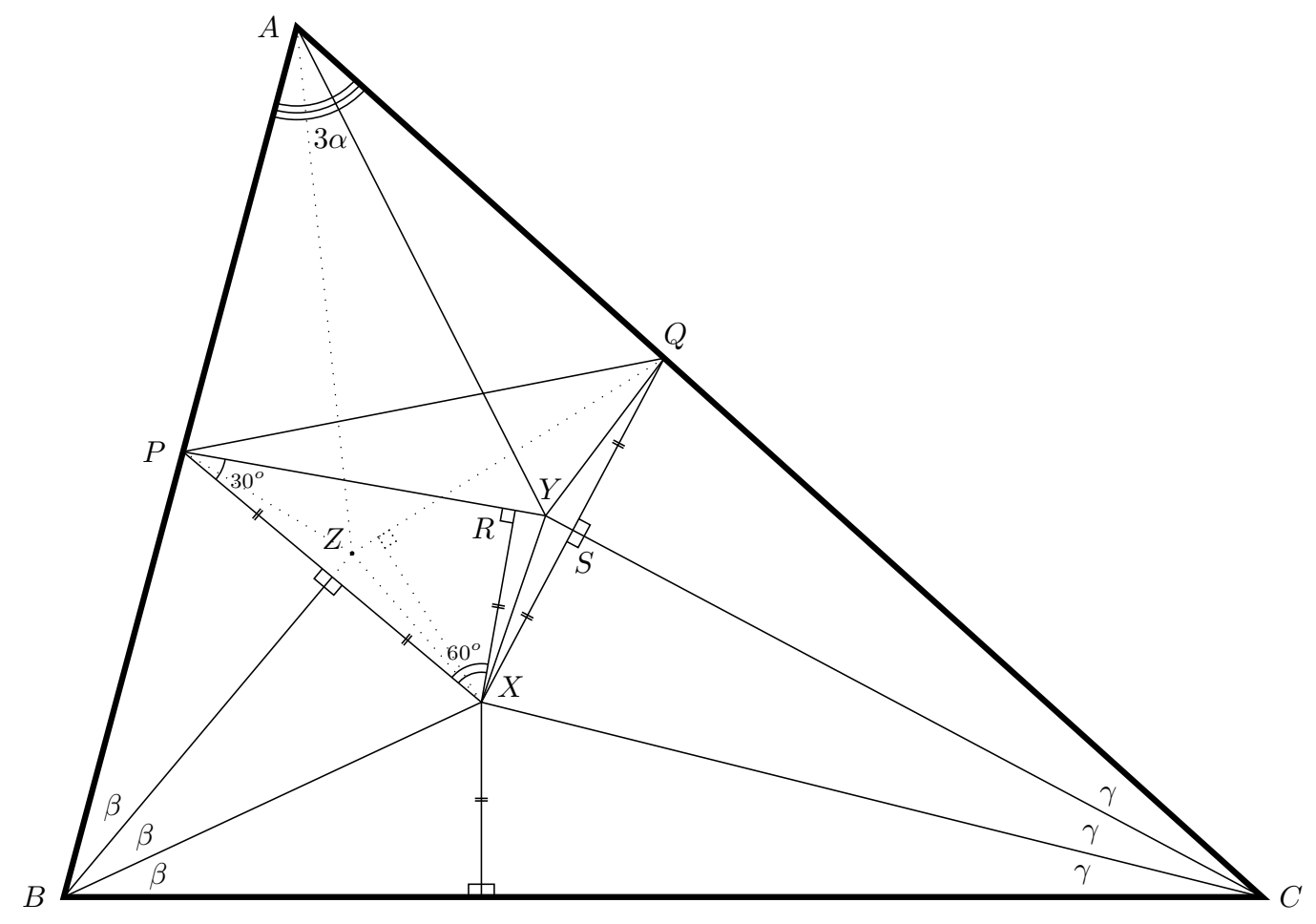

Now $\angle Q X P=360^{\circ}-2\left(90^{\circ}-\beta\right)-2\left(90^{\circ}-\gamma\right)=120^{\circ}-2 \alpha$. So $\angle Y X R=\angle S X Y=\angle Y Q S=\frac{1}{2}\left(\angle Q X P-60^{\circ}\right)=30^{\circ}-\alpha$. As $\triangle P Q X$ is isosceles its base angles $\angle X P Q$ and $\angle P Q X$ are both $30^{\circ}+\alpha$ so $\angle Y P Q=\alpha$ and $\angle P Q Y=\left(30^{\circ}+\alpha\right)-\left(30^{\circ}-\alpha\right)=2 \alpha$. 
Therefore $\angle Q Y P=180^{\circ}-3 \alpha$. And now for the advanced bit. Finn spots this is supplementary to $\angle B A C$ making $A P Y Q$ a cyclic quadrilateral. Consequently $\angle Y A Q=\angle Y P Q=\alpha$ which fixes $Y$ as the Morley vertex adjacent to $A C$. Next he performs a similar construction (shown in outline) starting from a right-angled triangle on hypotenuse $Q X$ and angles $30^{\circ}$ and $60^{\circ}$ at $Q$ and $X$ respectively. This generates three more wedges which are clearly congruent to the first three, plus the Morley vertex $Z$ adjacent to $A B$. In particular $|X Y|=|X Z|$ and $\angle Y X Z=60^{\circ}$ from which he deduces that $\triangle X Y Z$ is equilateral.

\section{Conclusion}

After scanning numerous proofs the only "modern" one I've seen that is remotely like this is given in [2] and attributed to W. E. Philip. William Edward Philip was Third Wrangler at Cambridge in 1894, but despite many references to [2] in the literature the beauty of his proof seems to have been strangely overlooked. Indeed [2] also contains a version of Leon Bankoff's 1962 trigonometric proof, long regarded as the easiest non-backward approach to the theorem. As years passed without anyone finding a short, simple, non-backward geometric proof a mistaken belief has proliferated that no such proof exists. So, as the centenary of its publication approaches, the time seems ripe to call attention to [2] and bring it back centre stage.

\section{REFERENCES}

[1] A. Bogomolny: http://www.cut-the-knot.org/triangle/Morley/index.shtml, Morley's Miracle from Interactive Mathematics Miscellany and Puzzles

[2] F. Glanville Taylor \& W. L. Marr: The six trisectors of each of the angles of a triangle, Proc. Edin. Math. Soc. 32 (1914), 119-131

[3] M. R. F. Smyth: MacCool's proof of Morley's Miracle, Irish Math. Soc. Bulletin 63 (2009), 63-66

Roger Smyth was a research student of Trevor West and received his Ph.D from Dublin University in 1972. Thereafter he moved into Information Technology and worked in Queen's University Belfast and the Northern Ireland Department of Health. His primary line is Fredholm theory in Banach algebras, but he maintains a recreational interest in simple Euclidean geometry.

(Roger Smyth) 15 Harberton Avenue, Belfast

E-mail address: malcolm.smyth@ntlworld.com 